\title{
Global Rotational Properties of Barred Galaxies
}

\author{
A. Bosma \\ Observatoire de Marseille, 2 Place Le Verrier, 13248 Marseille Cedex 4 , \\ France
}

\begin{abstract}
A general overview is presented of the global gas kinematics of barred spirals, using $\mathrm{HI}$ and $\mathrm{H} \alpha$ data. The question of dark matter in barred spirals is also discussed.
\end{abstract}

\section{Introduction}

The determination of the global gas kinematics of barred spirals is much more difficult than that of ordinary spirals. This is due to the very inhomogeneous distribution of the main kinematic tracers, ionized and neutral hydrogen gas. In general, the HI data provide at best a global rotation curve, while the $\mathrm{H} \alpha$ data provide at best an estimate of the amount of peculiar motions directly attributable to the action of the bar. Only for a few galaxies is complete kinematical information available, but this might rapidly change in the near future.

Already in the 1970 s there were sufficient data on the global velocity fields of spiral galaxies to demonstrate several main characteristic deviations from circular motion implying pure rotation. The two major bi-symmetric distortions are 1) oval or bar distortions, for which the kinematical major and minor axes are not perpendicular, and 2) kinematical warps, for which the kinematical axes stay perpendicular (cf. Bosma 1978, 1981). As it is possible to imagine a warped barred spiral, sometimes the distinction is not clearcut. If the distortions are in the inner parts, I tend to favor the oval distortion interpretation. This is based partly on the absence of strong optical warps in most unperturbed edge-on galaxies. As is clear from the literature, even today there are suggestions based on velocity fields for strong warps within the optical image, where a simpler interpretation can be had if galaxies are thought to be planar and barred.

For this review, it seems useful to distinguish categories of galaxies according to their main characteristic interest: dust lanes, inner rings, outer rings, etc., to see what the major results are.

\section{Characteristics of the Velocity Fields}

\subsection{SBb Galaxies With Dust Lanes}

The primary aim here is to test the prediction that the dust lanes constitute the loci of shocks (cf. Prendergast 1962), and to determine from detailed modelling some of the quantities not directly observable such as the pattern speed of the bar. In the late seventies the advances in numerical computations went hand 
in hand with detailed observations. In particular, the galaxy NGC 5383 served as a prototype. $\mathrm{H} \alpha$ data by Peterson et al. (1978) showed strong deviations from circular motions. Supplementary HI data by Sancisi, Allen \& Sullivan (1979) permitted to determine a rotation curve. Several detailed models were constructed for this galaxy (e.g. Huntley 1978, Sanders \& Tubbs 1980, Duval \& Athanassoula 1983), in which the main characteristics of the non-circular motions could be reproduced. These reveal themselves by a skewing of the isovelocity contours around the systemic velocity towards the bar, and a corresponding S-shape distortion in the kinematical major axis. Unlike in warps, the kinematical minor axis is not perpendicular to the kinematical major axis. Of course, the amplitude of the non-circular motions does not only depend on the strength of the bar, but also on angular resolution and the viewing angle (cf. Figure 41 of Athanassoula 1984 for an illustration of the latter).

Further improvements in the gas flow codes (e.g. Van Albada 1985), as well as extensive calculations of the orbital families in the corresponding potentials, have lead to strict conditions for which shocks resembling the observed dust lanes can occur (Athanassoula 1992a,b). These include the realization that the presence of a $\mathrm{x}_{2}$ family of orbits is not only necessary, but that a sizeable extent is needed.

The more modern data are in good agreement with this picture. Radio continuum emission is clearly enhanced in the dust lanes of M83 and NGC 1097 (Ondrechen \& Van der Hulst 1983, Ondrechen 1985), thus corroborating the notion that dust lanes are the loci of compressed gas. Long slit emission line spectroscopy along directions perpendicular to the dust lanes show, in a few cases, the expected velocity jumps (Pence \& Blackman 1982 for NGC 6221, and Lindblad \& Jörsäter 1988 for NGC 1365). And recent CO data show evidence for a strong concentration of molecular material in some dust lanes (e.g. Handa et al. 1990). However, when the shock velocities are very high, the amount of shear is so large that molecular clouds would not have the time to assemble (cf. Tubbs 1982, Athanassoula 1992b). This might explain the large variety in appearance of the dust lanes and OB-associations: the absence of the latter could just indicate the regions of high shear so that the dust lanes are there, but not the molecular gas and their associated regions of star formation.

A few other $\mathrm{SBb}$ galaxies have now been mapped in HI. The best studied galaxy is NGC 1365, for which both a $\mathrm{H} \alpha$ velocity field (Teuben et al. 1986) and detailed HI studies are available (Ondrechen \& Van der Hulst 1989, Jörsäter \& Van Moorsel 1995). The data by Teuben et al. (1986) clearly indicate that an inner Lindblad resonance is present in this galaxy, in agreement with the gas flow studies. The most recent modelling effort has been done by Lindblad et al. (1995, also Lindblad, these proccedings).

NGC 1097 has been studied by Ondrechen et al. (1989), who find that the HI emission is concentrated strongly in the outer arms, and is weak in the bar region. The velocity field indicates deviations from circular motions in the bar region, as in NGC 5383, as well as strong local deviations associated with the outer arms. Note that the continuum emission shown by Ondrechen \& Van der Hulst (1983) indicates not only shock regions in the straight dust lanes leading the bar, but, at least at the SE side, also at the edge of the bar making a sharp angle with the main dust lane. This behavior of the shocks can also be seen in some of the simulations by Athanassoula (1992b). Unfortunately, the presence 
of a companion perturbs the outer disk of this galaxy, hence no detailed model has yet been attempted.

Another study concerns NGC 1300 (England 1989a, 1989b), who finds that the HI here also is very strongly concentrated in the arms. He attempts to model his data and finds reasonable qualitative agreement using a bar model, but a quantitative comparison remains unsatisfactory.

\subsection{SBb Galaxies With Inner Rings}

These galaxies, such as NGC 1398, NGC 3351, etc. are less well studied, in part because the bar itself is devoid of $\mathrm{H} \alpha$-emission. Moore \& Gottesman (1995) studied NGC 1398, and find that there is no emission in the central parts, including the bar region. Only the region comprising the inner ring and the outer pseudo-ring contains HI emission. They attempt to derive a pattern speed from the rotation curve data, but do not obtain a unique answer.

Gottesman et al. (1984) studied NGC 3992, and find no HI emission in the central parts including the bar, although there is normal HI emission in the disk. The velocity field does show the expected deviations from circular motion. A modelling attempt has been reported in Hunter et al. (1988).

Broeils (1992) presents a HI study of NGC 6674, amidst several studies of ordinary spiral galaxies. The bar in this galaxy seems relatively weak, although there is some sign of non-circular motion due to the bar.

Buta, in a series of papers devoted to barred galaxies with rings, discusses $\mathrm{H} \alpha$ kinematics from Fabry-Perot interferometry for NGC 1433 (Buta 1986), NGC 7531 (Buta 1987a), NGC 6300 (Buta 1987b), and NGC's 1512, 3351, 4725 and 4736 (Buta 1988). Although these data in general show the expected deviations from circular motions associated with the presence of a bar or oval, the data are often limited to the inner parts, and particularly the center and the inner ring. Such data merit to be complemented with HI data and surface photometry for a more detailed study. For several galaxies this is now underway.

\subsection{SA Galaxies With (Outer) (Pseudo-) Rings and Oval Distortions}

In the late 70's a few galaxies, while not classified as barred spirals, were identified as having oval distortions, based on distinct signatures in their velocity fields (cf. Bosma 1978, 1981). Typical examples are NGC 4151 (Bosma et al. 1977a), NGC 4258 (Van Albada 1980) and NGC 4736 (Bosma et al. 1977b).

The new VLA B-array 21-cm HI data of NGC 4151 shown by Shone et al. (these proceedings) fully confirm the main result from previous work by Davies (1973), Bosma et al. (1977a) and Pedlar et al. (1992), i.e. that the main body of this galaxy is a fat bar. Note that Boksenberg et al. (1995) find from HST data evidence for a central mass concentration of about $10^{9} \mathrm{M}_{\odot}$ in the inner few arcsec. Compared to the total mass of $210^{11} \mathrm{M}_{\odot}\left(\mathrm{H}_{0}=50 \mathrm{~km}\right.$ $\mathrm{s}^{-1} \mathrm{Mpc}^{-1}$ ) enclosed within the outer ring radius, this means that a central mass concentration of $0.5 \%$ has not managed to scatter the $x_{1}$-orbits of the bar sufficiently to decay it. However, if only the region of the fat bar is considered, the fraction of the central mass concentration to the mass enclosed within the bar radius becomes about $1 \%$. If only the disk mass out to this radius is considered, this fraction might become of order $2 \%$. This is of some interest in view of 
theoretical notions that bars might decay due to the build up of a central mass concentration of only a few percent (e.g. Hasan \& Norman 1990, Friedli 1994).

NGC 4736 has been well studied, and is a prime example of a complex galaxy where several phenomena can be noticed at once. The early HI data (Bosma et al. 1977b) showed HI in an inner ring, an outer ring, and in the main body in between. From the kinematics, this main body was concluded to have an oval shape. More recent data have by and large confirmed this picture (Mulder \& Van Driel 1993), but infrared data have revealed a bar inside the inner ring (cf. Möllenhoff, Matthias \& Gerhard 1995). It thus appears that this galaxy has two pattern speeds, one associated with the inner bar, and the other one with the oval. A similar situation exists for NGC 1068 (cf. Bosma 1992).

The data for NGC 4258 (Van Albada 1980) show strong indications for the presence of a bar, and the outer arms could then be thought of as a pseudoring. This is most clearly visible in the image of the HI distribution. The kinematics show all the signs for the presence of a bar. The interpretation for this galaxy has been obscured somewhat by the issue of the anomalous arms, which are not detected in $\mathrm{HI}$, but which are strong in the radio continuum, in $\mathrm{H} \alpha$, and also now in CO (Krause et al. 1990). For a recent account of the spiral structure in this galaxy, see Courtes et al. (1993). Evidence for a central black hole in this galaxy has been presented by Miyoshi et al. (1995).

Another galaxy in this category is NGC 1808, which has been studied by Koribalski et al. (1993). Even though the appearance of this galaxy, with a fat bar containing spiral filaments, and two outer arms forming a pseudo ring, makes this a good case for a $\Theta$-galaxy (in other words, an oval distortion), these authors proceed to construct a tilted ring warp model to their data. However, the higher angular resolution (but poorer sensitivity) HI data by Saikia et al. (1990) quite clearly show that there are significant non-circular motions entirely consistent with the idea of the main body of this galaxy being a fat bar.

Finally, there is the case of NGC 7217, discussed in detail by VerdesMontenegro et al. (1995) and Buta et al. (1995). The HI is predominantly in an outer ring, but there is little deviation from axial symmetry, and no clearcut bar has been found in optical images. Despite of this, the simultaneous presence of three rings (a nuclear ring, an inner ring and the outer ring) can be accounted for with a single pattern speed of $86 \mathrm{~km} \mathrm{~s}^{-1} \mathrm{kpc}^{-1}$. Athanassoula (these proceedings) describes scenarios in which the bar in this galaxy could have decayed, while the rings still persist.

\subsection{SB0-b Galaxies With Outer Rings}

An extensive HI study of early type disk galaxies has been made by Van Driel (1987, cf. Van Driel \& Van Woerden 1991 for a summary of the main conclusions). A number of these galaxies are barred spiral galaxies with outer rings, where in most cases the HI is almost absent from the bar region, and concentrated in the outer ring, although in a number of cases there is extended HI beyond it.

The data on true SB0 galaxies show little relation between the HI emission and the main optical image: usually the HI is found outside the optical disk in an annulus, but the kinematics indicate that its spatial orientation is different from the main body. This is found for NGC 2787 (Shostak 1987), and NGC 
4262 (Krumm et al. 1985). Such a result is also found for several ordinary S0 galaxies (cf. Van Driel \& Van Woerden 1991). For the galaxy NGC 3941 (Van Driel \& Van Woerden 1989), however, the outer HI ring is more or less in the same plane as the optical disk.

For slightly later type disk galaxies the situation is different, since here most of the HI does coincide with an outer optical ring. Van Driel, Rots \& Van Woerden (1988) studied the SB0/a galaxies NGC 1291 and NGC 5101. Little or no HI emission is found in the bar region itself. Note that NGC 1291 has strong X-ray emission in the central parts (Bregman, Hogg \& Roberts 1995). This galaxy is seen very face-on, and the $\mathrm{HI}$ is mainly concentrated in the outer ring, with some extended emission beyond it. The velocity field indicates that this outer HI is in a warped disk. The inner edge of the HI ring coincides with the inner edge of the outer ring seen in optical images. For NGC 5101 the results are rather similar, with extended $\mathrm{HI}$ emission beyond the optical ring. Here the velocity field is more regular, and a rotation curve could be derived (see below).

For the rather obscured SA(r)0/a galaxy NGC 7013 Knapp et al. (1984) find the HI emission mainly in two rings, and a velocity field which shows the characteristics of an oval distortion. They prefer to model this with circular rings, though with the inner ring having a different position angle from the disk and the outer ring. I suspect this galaxy to be a barred spiral.

Work on a few later type ringed SB galaxies has been reported by Buta \& Van Driel (1992). They considered the (R')SB(rs)ab galaxy NGC 2273, where again the HI is mainly in the outer ring and the rotation curve is flat, and the (R)SB(rs)bc galaxy NGC 6217. For this latter galaxy there is HI in the inner parts, mainly in the inner ring, and in the outer ring and beyond. The kinematical major axis does not coincide with the major axis of the outer ring, indicating a possible intrinsic elongation of the latter.

Schommer et al. (1988) present $\mathrm{H} \alpha$-kinematics for the Seyfert galaxy NGC 5728. They find S-distorted velocity contours, which are interpreted as due to the bar in this galaxy. In the central parts, however, the velocity data is more complicated.

\subsection{SBc Galaxies}

For late type $\mathrm{SBc}$ galaxies, a few studies are available, which show that there is HI present in the bar. Data for NGC 1073 (England et al. 1990) show that there is $\mathrm{HI}$ gas in the bar, and the kinematics indicate that the bar is within the rising part of the rotation curve. For NGC 4731, Gottesman et al. (1984) find the $\mathrm{HI}$ emission strongly concentrated in the spiral arms, although there is also gas in the bar region. The rotation curve is still rising beyond the bar radius, but no serious attempt has been made to model this galaxy in detail. For NGC 925 the best data can be found in the survey of Wevers et al. (1986). Contrary to NGC 4731 , the bar is very weak, and does not seem to have much influence on the kinematics of the gas in the inner parts.

Ball (1986) has studied the SBc galaxy NGC 3359. Here again there is HI in the bar although not very prominent, and further out in the disk. The bar is short and rather weak, and extends roughly out to the solid body part of the rotation curve. There are large scale deviations from circular motion. In a modelling effort, Ball (1992) describes difficulties to achieve a realistic model 
only with the observed bar taken into account: a more extended bar/oval would be needed to describe the deviations in the velocity field and the presence of the stronger spiral arms.

Duval et al. (1991) report a detailed $\mathrm{H} \alpha$ study of NGC 7741, and fit their data with a simplified bar model. They find a bar-to-disk ratio, inside the bar radius, of about $30 \%$.

Pence (1981) studied the galaxy NGC 253 in $\mathrm{H} \alpha$, and finds indications for the presence of a bar. This bar has been seen in the near infrared by Scoville et al. (1985). For the most recent data on this galaxy, see Arnaboldi et al. (1995).

\section{6. $\mathrm{SBm}$ - IBm Galaxies}

For these galaxies, the bar may not be in the center of the disk, but offset to one side, like in the Large Magellanic Cloud. This has been described extensively in a review by De Vaucouleurs \& Freeman (1972), and models of the gas flow in such a situation have been computed by Colin \& Athanassoula (1989). It is thought that the gas streaming causes a pile up of gas at one end of the bar, leading to a large region of star formation (e.g. like 30 Doradus in the LMC).

For a few of these galaxies detailed HI or $\mathrm{H} \alpha$ studies have become available, and the above description, while valid in some cases, is not upheld in others. E.g. NGC 2366 (Wevers et al. 1986) has a regular bi-symmetric velocity field. For NGC 1313, the H $\alpha$-data from Marcelin \& Athanassoula (1982) indicated the possibility of an offset bar, but the HI-data from Peters et al. (1988) and the new AT data from Ryder et al. (1994) show otherwise.

For NGC 4027, a detailed $\mathrm{H} \alpha$ study by Pence et al. (1988) was complemented by a HI study by Phookun et al. (1992). Contrary to expectation, the center of symmetry of the $\mathrm{H} \alpha$ velocity field coincides closely with the center of the bar. The one-armed appearance of NGC 4027 seems to be the result of strong interaction with the companion NGC $4027 \mathrm{~A}$.

NGC 4449 (Van Woerden et al. 1974) and IC 10 (Cohen 1979, Shostak \& Skillman 1989) remain the galaxies for which velocity reversals are seen: the III halo has an altogether different velocity field than the inner parts, indicating that these galaxies have not yet settled into a stable structure.

\section{Dark Matter in Barred Spiral Galaxies}

The extended HI rotation curves of ordinary spirals, which do not show any Keplerian drop-off in the outer parts, constitute the most solid evidence for the presence of dark matter. For recent reviews on this subject, see e.g. Bosma. (1995) and Sackett (1995). Here I will consider the evidence for dark matter in barred spirals, to see whether there is really a difference in their dark matter content compared to ordinary spirals. It should be remarked from the outset that, due to the inhomogeneous nature of the HI and $\mathrm{H} \alpha$ emission in barred spirals, their rotation curves are not determined with the same accuracy as those for ordinary spirals. 


\subsection{Should There Be Dark Matter in Barred Spirals?}

This question is more frequently asked than it deserves to be. Indeed, the notion is sometimes held that barred spirals are the result of the bar instability in disk galaxies (which could well be true), and therefore should not have a dark halo (which is not necessarily true). This mistake arises from misunderstanding one aspect of the historical development of the dark matter problem: initially, a dark halo was proposed to help cure the bar instability in disks. However, what matters here is the dark matter in the halo acting on the disk. Any dynamically hot component inside the disk radius will help, but any halo mass outside the disk radius will not contribute. Thus the stabilizing influence of a bulge should not be underestimated. Likewise, the presence of a hot disk (perhaps dark) may help to cure the bar instability (cf. Ostriker \& Peebles 1973, Athanassoula \& Sellwood 1986).

\subsection{The Dynamics of the Satellites of NGC 3992}

Gottesman \& Hunter (1982) obtained HI detections of three dwarf companions in the field of the SBb(r) galaxy NGC 3992, and proceeded to calculate the mass of this galaxy using a mass estimator based on the relative velocities of the three satellites with the main system. They found that little additional mass is needed beyond the outer radius of the HI detected in NGC 3992, if the mass estimate is to be taken seriously. The statistical uncertainties associated with such a small number of satellites are rather large. Erickson et al. (1987) have made an analysis of several more groups, and come again to the conclusion that the haloes of galaxies are not that massive. However, for a larger sample of unbarred $\mathrm{Sb}$ and Sc galaxies, Zaritsky \& White (1994) do find evidence for massive and extended dark haloes. It seems that the result for NGC 3992 is just a bit odd, based on the application of a statistic on a single object. In any case, in the modelling described by Gottesman et al. (1984) and Hunter et al. (1988) the possibility of a dark halo component is taken into account.

\subsection{The Behavior of the Rotation Curves}

In the early compilation of rotation curves in Bosma $(1978,1981)$ the few barred or oval galaxies do not stand out in the behavior of their rotation curves: they are about as flat as the others, and, if all the mass is in the disk, local massto-light ratios become very high in the outer parts (cf. Bosma \& Van der Kruit 1979). This concerns the oval galaxies NGC 4151, NGC 4258 and NGC 4736 , and the barred spirals NGC 5383 and M83. I have checked the literature to see whether the shape of the rotation curves of barred spirals observed more recently really differ from those observed for ordinary spirals. In most cases there are no noticeable differences, and the predominant characteristic is that most rotation curves are rising, flat or slightly declining, just like the rotation curves of ordinary galaxies, and in most of the modelling work a dark halo component has been included. For a few objects, in particular NGC 3992 (Gottesman et al. 1984) and NGC 1073 (England et al. 1990), the rotation curve might be abruptly declining in the outer parts, but the uncertainties are rather large due to a poor signal-to-noise ratio. On the other hand, the extreme warp around M83 outlined by Rogstad et al. (1974) suggests the occasional presence of an extended HI envelope also around barred spirals. 
One galaxy qualifies as a good candidate for not needing a dark halo: this is the galaxy NGC 5101, observed by Van Driel, Rots \& Van Woerden (1988). This RSB(rs)0/a galaxy is seen rather face-on, and the HI is distributed mainly in the outer ring and beyond that. The velocity field is rather regular, and the rotation curve can be derived, although the inclination correction is rather uncertain. In any case, the rotation curve declines, and this decline can be fitted with an exponential disk which has a scalelength corresponding to the optical scalelength derived from the $B$-band photometry given in Lauberts \& Valentijn (1989). However, it should be remarked that outer rings tend to be blue, and that if this is so then the $B$-band scalelength overestimates the scalelength of the bulk of the mass in the disk. A $K$-band image of NGC 5101 seems needed to assess whether the rotation data for NGC 5101 really can be fitted without the need for a dark halo. Van Driel et al. (1988) remark that a warp of only $8^{\circ}$ in the outer HI disk is needed to keep the rotation curve flat.

Jörsäter \& Van Moorsel (1995, see also Jörsäter, these proceedings) argue for a Keplerian decline of the rotation curve in NGC 1365. To achieve this, they propose that the inclination of the galaxy increases drastically from $40^{\circ}$ to $55^{\circ}$ over the outer third of the optical disk. Such a large warp within the optical radius is seldom seen in other galaxies (cf. Sanchez-Saavedra et al. 1990), and one can only imagine how odd this galaxy will look if the inner parts are seen edge-on. In view of the presence of non-circular motions due to the bar, it is not at all clear that their treatment of the two-dimensional velocity field is correct. If no warp is assumed, the rotation curve declines much less, and the conclusions about the dark matter in NGC 1365 would be very different.

\subsection{The Small, Late Type Galaxies}

Odewahn (these proceedings) discusses an interesting relation between the frequency of barred spirals and the amplitude of the rotation curve as estimated from the $21-\mathrm{cm}$ line velocity width. Though giant galaxies $\left(V_{\max }\right.$ larger then about $160 \mathrm{~km} \mathrm{~s}^{-1}$ ) are classified in equal percentages as $\mathrm{SA}, \mathrm{SAB}$ or $\mathrm{SB}$, the fraction of galaxies classified SB increases smoothly to about $80 \%$ as the maximum rotational velocity decreases to about $40 \mathrm{~km} \mathrm{~s}^{-1}$. Since dwarf galaxies are dark halo dominated, like e.g. DDO 154 (Carignan \& Freeman 1988), this increase in the frequency with which small galaxies are classified as barred is intriguing.

Several explanations come to mind. Of course it could be said that the classifier "sees" more bars, and/or that the bars in these systems are not very massive anyway, and thus insignificant with respect to the disk. However, the statistics of the axial ratios of very late type galaxies show that a fair fraction of the magellanic irregulars are much thicker than the disks of late type spirals (cf. Binney and De Vaucouleurs 1981, Bosma 1994). Hence, if these galaxies are dark matter dominated, the dark halo could dominate the shape of the optical image completely. Since the natural shape of a halo might be triaxial, the optical image should then be triaxial to a certain extent. For larger galaxies, the disk might be sufficiently strong so that its dissipative formation did "rounden" out the halo (cf. Dubinski 1994). 


\section{Concluding Remarks}

As function of Hubble type, the HI distribution in barred disk galaxies thus shows a progression, in the sense that for early Hubble types, SB0/a - SBa, the $\mathrm{HI}$ is predominantly distributed in the outer ring and sometimes beyond, for intermediate types, $\mathrm{SBb}$, the $\mathrm{HI}$ is frequently absent from the central parts, but otherwise distributed throughout the disk, while for $\mathrm{SBc}$ and later there is also $\mathrm{HI}$ in the central parts. The rotation data are in general consistent with the need for dark matter in and around barred spirals, just as for ordinary spirals.

It is clear that, despite the enormous observational effort sofar, only for a few barred spirals could detailed models be constructed which can be constrained successfully by the observations. A concerted approach involving several ways of tracing the kinematics, and multi-band surface photometry, might improve this situation.

Acknowledgments. I would like to thank Lia Athanassoula for frequent discussions about barred galaxies in general, and several aspects of this review in particular.

\section{References}

Arnaboldi, M., Capaccioli, M., Cappellaro, E., Held, E. V., Koribalski, B. 1995, AJ, 110,199

Athanassoula, E. 1984, Phys. Reports, 114, 319

Athanassoula, E. 1992a, MNRAS, 259, 328

Athanassoula, E. 1992b, MNRAS, 259, 345

Athanassoula, E. \& Sellwood, J. A. 1986, MNRAS, 221, 213

Ball, R. 1986, ApJ, 307, 453

Ball, R. 1992, ApJ, 395, 418

Boksenberg, A. et al. 1995, ApJ, 440, 151

Bosma, A. 1978, Ph. D. thesis, University of Groningen

Bosma, A. 1981, AJ, 86, 1825

Bosma, A. 1992, in Morphological and Physical Classification of Galaxies, G. Longo et al., Dordrecht: Reidel, 207

Bosma, A. 1994, in Dwarf Galaxies, G. Meylan \& P. Prugniel, Garching-beiMünchen: ESO, 187

Bosma, A. 1995, in Dark Matter, S. S. Holt \& C. L. Bennett, New York: AIP, in press

Bosma, A., Ekers, R. D., \& Lequeux, J. 1977a, A\&A, 57, 97

Bosma, A., Van der Hulst, J. M., \& Sullivan III, W. T. 1997b, A\&A, 57, 373

Bosma, A. \& Van der Kruit, P. C. 1979, A\&A, 79, 281

Bregman, J. N., Hogg, D. E., \& Roberts, M. S. 1995, ApJ, 441, 561

Broeils, A. H. 1992, Ph. D. thesis, University of Groningen

Buta, R. 1986, ApJS, 61, 631

Buta, R. 1987a, ApJS, 64, 1 
Buta, R. 1987b, ApJS, 64, 383

Buta, R. 1988, ApJS, 66, 233

Buta, R. et al. 1995, ApJ, 450, 593

Carignan, C. \& Freeman, K. C. 1988, ApJ, 332, L33

Cohen, R. J. 1979, MNRAS, 187, 839

Colin, J. \& Athanassoula, E. 1989, A\&A, 214, 99

Courtes, G. et al. 1993, A\&A, 268, 419

Davies, R. D. 1973, MNRAS, 161, 25P

de Vaucouleurs, G. \& Freeman, K. C. 1973, Vistas in Astronomy, 14, 163

Dubinski, J. 1994, ApJ, 431, 617

Duval, M. F. \& Athanassoula, E. 1983, A\&A, 121, 297

Duval, M. F. et al. 1991, A\&A, 241, 375

England, M. 1989a, ApJ, 337, 191

England, M. 1989b ApJ, 344, 669

England, M., Gottesman, S. T., \& Hunter, J. H. 1990, ApJ, 348, 456

Erickson, L. K., Gottesman, S. T., \& Hunter, J. H. 1987, Nature, 325, 779

Friedli, D. 1994, in Mass-Transfer Induced Activity in Galaxies, I. Shlosman, Dordrecht: Kluwer, 268

Gottesman, S. T. \& Hunter, J. H. 1982, ApJ, 260, 65

Gottesman, S. T., Ball, R., Hunter, J. H., \& Huntley, J. M. 1984, ApJ, 286, 471

Handa T. et al. 1990, PASJ, 42, 1

Hasan, H. \& Norman, C. A. 1990, ApJ, 361, 69

Hunter, J. H. et al. 1988, ApJ, 324, 721

Huntley, J. M. 1978, ApJ, 225, L101

Jörsäter, S. \& Van Moorsel, G. A. 1995, A\&A, 110, 2037

Knapp, G. R., Van Driel, W., Schwarz, U. J., Van Woerden, H., \& Gallagher, J. S. 1984, A\&A, 133,127

Koribalski, B., Dahlem, M., Mebold, U., \& Brinks, E. 1993, A\&A, 268, 14

Krause, M., Cox, P., Garcia-Barreto, J. A., \& Downes, D. 1990, A\&A, 233, L1

Krumm, N., van Driel, W., \& van Woerden, H. 1985, A\&A, 144, 202

Lauberts, A. \& Valentijn, E. A. 1989, The Surface Photometry Catalog of the ESO-Uppsala Galaxies, Garching-bei-München: ESO

Lindblad, P. O. \& Jörsäter, S. 1988, in Evolution of Galaxies, proc of the 10th European Regional I.A.U. meeting, J. Palouš, 289

Lindblad, P. A. B., Lindblad, P. O., \& Athanassoula, E. 1995, A\&A, submitted Marcelin, M. \& Athanassoula, E. 1982, A\&A, 105, 76

Miyoshi et al. 1995, Nature, 373, 127

Möllenhoff, C., Matthias, M., \& Gerhard, O. E. 1995, A\&A, 301, 359

Moore, E. M. \& Gottesman, S. T. 1995, ApJ, 447, 159

Mulder, P. S. \& Van Driel, W. 1993, A\&A, 272, 63

Ondrechen, M. P. 1985, AJ, 90, 1474.

Ondrechen, M. P. \& Van der Hulst, J. M. 1983, ApJ, 269, L47. 
Ondrechen, M. P. \& Van der Hulst, J. M. 1989, ApJ, 342, 29.

Ondrechen, M. P., Van der Hulst, J. M., \& Hummel, E. 1989, ApJ, 342, 39.

Ostriker, J. P. \& Peebles, P. J. E. 1973, ApJ, 186, 467.

Pedlar, A., Howley, P., Axon, D. J., \& Unger, S. W. 1992, MNRAS, 259, 369

Pence, W. D. 1981, ApJ, 247, 473

Pence, W. D. \& Blackman, C. P. 1984, MNRAS, 207, 9.

Pence, W. D. et al. 1988, ApJ, 326, 564

Peters, W. et al. 1994, MNRAS, 269, 1025

Peterson, C. J., Rubin, V. C., Ford, W. K., \& Thonnard, N. 1978, ApJ, 219, 31

Phookun, B., Mundy, L., Teuben, P. J., \& Wainscoat, R. J. 1992, ApJ, 400, 516

Prendergast, K. H. 1962, unpublished

Rogstad, D. H., Lockhart, I. A., \& Wright, M. C. H. 1974, ApJ, 193, 309

Ryder, S. et al. 1995, AJ, 109, 1592

Sackett, P. D. 1995, in Gravitational Lensing, I.A.U. Symp. 173, in press

Saikia, D. J. et al. 1990, MNRAS, 245, 397

Sanchez-Saavedra, M. L., Battaner, E., \& Florido, E. 1990, MNRAS, 246, 458

Sancisi, R., Allen, R. J., \& Sullivan III, W. T. 1979, A\&A, 78, 217

Sanders, R. H. \& Tubbs, A. D. 1980, ApJ, 235, 803.

Schommer, R. A. et al. 1988, ApJ, 324, 154

Scoville, N. Z. et al. 1985, ApJ, 289, 129

Shostak, G. S. 1987, A\&A, 175, 4

Shostak, G. S. \& Skillman, E. 1989, A\&A, 214, 33

Teuben, P. J., Sanders, R. H., Atherton, P. D., \& Van Albada, G. D. 1986, MNRAS, 221, 1

Tubbs, A. D. 1982, ApJ, 255, 458

Van Albada, G. D. 1980, A\&A, 90, 123

Van Albada, G. D. 1985, A\&A, 142, 491

van Driel, W. 1987, Ph. D. thesis, University of Groningen

Van Driel, W. \& Buta, R. J. 1991, A\&A, 245, 7

Van Driel, W., Rots, A. H., \& Van Woerden, H. 1988, A\&A, 204, 39

Van Driel, W. \& Van Woerden, H. 1989, A\&A, 225, 317

Van Driel, W. \& Van Woerden, H. 1991, A\&A, 243, 71

Van Woerden, H., Bosma, A., \& Mebold, U. 1974, in La Dynamique des Galaxies Spirales, CNRS Coll. 241, L. Weliachew, 483

Verdes-Montenegro, L., Bosma, A., \& Athanassoula, E. 1995, A\&A, 300, 65

Wevers, B. H. M. R., Van der Kruit, P. C., \& Allen, R. J. 1986, A\&AS, 66, 505

Zaritsky, D. \& White, S. D. M. 1994, ApJ, 435, 599 